\title{
Ferromagnetic Glass-Coated Microwires for Electromagnetic Shielding for Medical Application
}

\section{Baranov $\mathrm{SA}^{1,2 *}$ \\ ${ }^{1}$ Institute of Applied Physics, Republic of Moldova, Russia \\ ${ }^{2}$ Shevchenko Pridnestrov'e State University, Republic of Moldova, Russia}

*Corresponding author: Serghei Alexei Baranov, Institute of Applied Physics, Academiei str.

\section{Mini Review}

Volume 4 Issue 2

Received Date: September 28, 2020

Published Date: October 19, 2020

5, Chisinau, MD 2028 Republic of Moldova, Russia, Tel: +(37322)226386; +(37322)731725;

Fax: +(37322)731725; Email: sabaranov07@mail.ru

\section{Abstract}

The absorption properties of radio absorption shielding made of a glass-coated amorphous magnetic micro- and nanowires have been studied by radionoise suppression experiments. The absorption parameter values observed are of $5 \mathrm{~dB}$ at $10 \mathrm{GHz}$. The losses profile is ascribed to the presence of natural ferromagnetic resonance in microwires.

Keywords: Glass-coated amorphous magnetic micro and nanowires; Magnetostriction; Magnetoelastic anisotropy; Natural ferromagnetic resonance; Noise

\section{Introduction}

The increasingly miniaturized devices at high frequencies lead to noise in medical apparatus. This fact has strongly promoted the development for noise suppression purposes [1-3]. As this research has evolved, the need to control the magnetic anisotropy in components came into attention since the field absorption is related to natural ferromagnetic resonance (NFR) [4-7].

On the other hand it has been widely documented that glass-coated amorphous magnetic micro- and nanowires (GCAMNWs) possess very interesting high frequency properties due to the strong magnetoelastic anisotropy which is induced in the fabrication process [4]. By defining strictly the geometric characteristics and the alloy composition the medical norms of electromagnetic field can be accurately controlled. Furthermore, the high production rate and high permeability feature of GCAMNWs without any rigid substrates are advantageous for use as noise absorbers of electromagnetic field.
In this report, amorphous glass-coated microwire arrays in a sheet configuration are proposed for use as absorbers. High frequency characteristics of thin amorphous microwires will be presented and evaluated as radionoise absorbers and medical protection radio absorption shielding.

During the manufacturing the GCAMNWs using the Ulitovsky-Taylor method the soften glass capillary is filled with a melted metal alloy with a further quenching [4].

The residual stresses, arising in metal core of GCAMNWs by during manufacturing, determine magnetic behavior of such GCAMNWs [4]. The metal is frozen into a very thin GCAMNWs. Thus a strong quenching stress (for example, $\sigma \sim 10^{9} \mathrm{~Pa}$ in ultrathin GCAMNWs) is introduced in the GCAMNWs [4-7].

\section{Radio-Absorption Shielding}

The designs of composites from GCAMNWs have following configurations. 


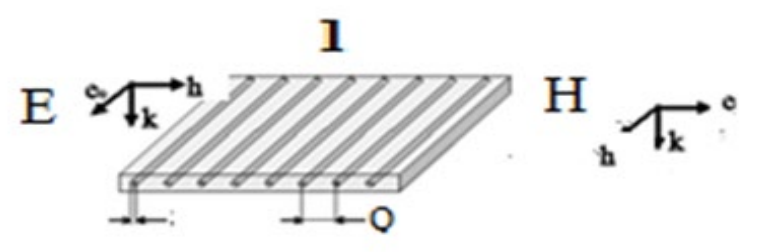

Figure 1a: Composite shielding for radio absorption protection with GCAMNWs where were marked in grating form. We can consider two types of orientation of a magnetic field: $\mathrm{E}$ and $\mathrm{H}$.

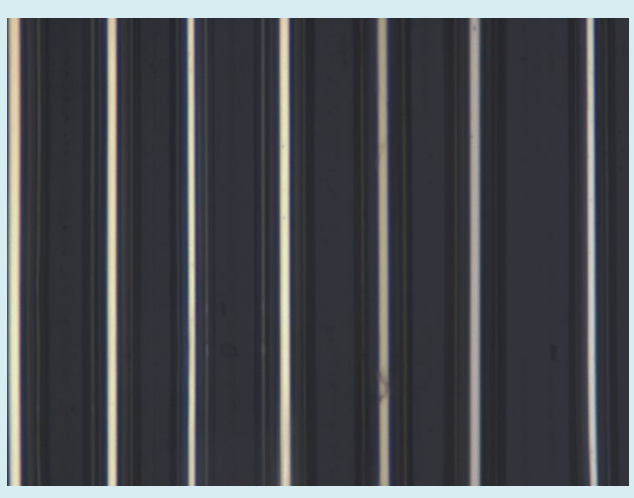

Figure 1b: Micrograph of an array of Co based microwires.

However, for very thin GCAMNWs with negative, positive and zero magnetostriction the domain structure is far more complicated due to a complex stress distribution within the small diameter. The simplest domain structure reported so far for these GCAMNWs is obtained in Baranov SA [8-10] (see also $[11,12])$.

As for the microwire array configuration for noise suppression experiments, 223 microwires $(10 \mu \mathrm{m}$ total diameter and length of $5 \mathrm{~mm}$ where the metal core diameter is $2 \mu \mathrm{m}$,) are parallel aligned (Figure $1 \mathrm{a}$ ) in an area of $5 \times 4$ $\mathrm{mm}^{2}$ as can be observed in Figure $1 \mathrm{~b}$. They are supported by a thin polymer sheet ( 100 $\mu$ m thickness).

\section{Noise Absorption Experiments}

In order to investigate the noise absorption performances a microstrip line (with $50 \Omega$ characteristic impedance) has been designed [1-3]. The signal line has a width of $610 \mu \mathrm{m}$ and thickness of $\mathrm{t}=35 \mu \mathrm{m}$ and is set on a 300 thick Teflon base with permittivity $\varepsilon_{\mathrm{r}}=3.6$. The insertion losses are estimated below 0.08 at $1 \mathrm{GHz}$. The performance of the line is measured using an Agilent E8363B network analyzer from 0.1 to $20 \mathrm{GHz}$. The microwire array was placed on the top of the signal line.
Samples of a single and twofold array of microwires were fabricated. The measured absorption coefficient $S_{21}$ on the microstrip line is showed in Baranov SA, Yamaguchi $\mathrm{M}$, Garcia $\mathrm{KL}$, Vazquez M [1]. The $S_{21}$ parameter at $1 \mathrm{GHz}$ decreases from the background of $-0.08 \mathrm{~dB}$ to $-0.154 \mathrm{~dB}$ and $-0.188 \mathrm{~dB}$ for the single layer and twofold layer (labeled as A to B). It is worth mentioning that larger decreases are observed at around $20 \mathrm{GHz}$. This is probably due to the metallic property of the wires.

The ratio of the power loss by the microwire layers to the input power calculated as $\mathrm{P}_{\text {loss }} / \mathrm{P}_{\text {in }}=1-\left(\left|\mathrm{S}_{11}\right|^{2}+\left|\mathrm{S}_{21}\right|^{2}\right)$ is shown in Figure 2 [1]. A loss peak nearby the resonance frequency is observed around for both samples. It is noted that the twofold sample fails to be effective correspondingly. The reason is that the second layer is not close enough to interact with the near field from the microstrip line.

From the experimental results described above the following matters should be considered for future improvements.

Although the amorphous wires exhibit superb frequency profiles and various advantages such as a high production rate and geometrical flexibility, the metal cores in the wires are coated with very thick glass layer resulting in a poor packing density of high permeability region in the wire array samples. If the packing density of 0.12 for the present samples were, for example, 0.8 , the power loss would be about 0.3 for a single layer. This is possible by removing the glass coating. Furthermore, by designing a wire array with proper conductivity and permeability, a high performance absorbers.

These issues will be treated intensively as this research work progresses in near future.

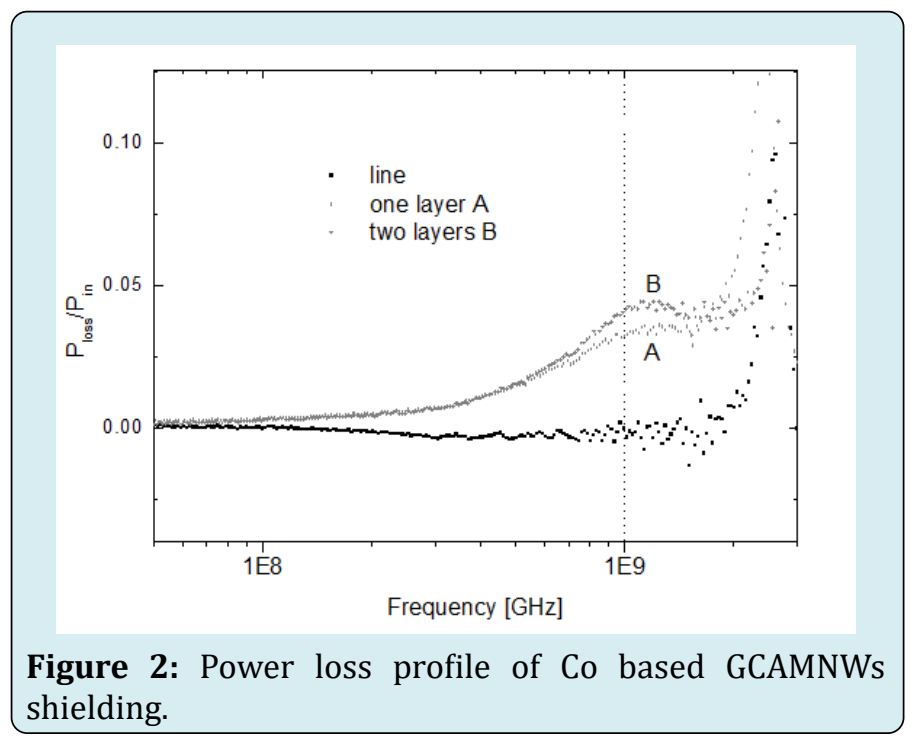


Physical Science \& Biophysics Journal

\section{Conclusion}

The high permeability features for very thin amorphous microwires for medical apparatus and HF radio absorption shielding were investigated. The electromagnetic noise absorption of sheet type samples with dimensions ( $5 \times 4$ ) $\mathrm{mm}^{2}$ made of 223 parallel aligned microwires was measured. The twofold layers exhibit of $-2.5 \mathrm{~dB}$, with insertion losses below 0.2 at $1 \mathrm{GHz}$ which corresponds to the resonance of magnetization at $1 \mathrm{GHz}$.

\section{References}

1. Baranov SA, Yamaguchi M, Garcia KL, Vazquez M (2008) Dimensional absorption high-frequency properties of the cast glass coated microwires. Surf Engin Appl Electrochem 44(6): 245-247.

2. Baranov SA, Yamaguchi M, Garcia KL, Vazquez M (2010) Application Of Amorphous Microwires for Electromagnetic Shielding. Moldavian J Phys Sci 9(1): 76-82.

3. Baranov SA (2016) New Perspective for Biomedical Productions: Application of Cast Amorphous Microwire for Electromagnetic Absorption. In: Sontea V, Tiginyanu I (Eds), $3^{\text {rd }}$ International Conference on Nanotechnologies and Biomedical Engineering. Vol 55, IFMBE Proceedings, Springer, Singapore, pp: 259-263.

4. Baranov SA, Larin VS, Torcunov AV (2017) Technology,
Preparation and Properties of the Cast Glass-Coated Magnetic Microwires. Crystals 7(6): 136.

5. Baranov SA (1998) Use of a microconductor with natural ferromagnetic resonance for radio-absorbing materials. Tech Phys Lett 24: 549-550.

6. Baranov SA (2015) Engineering Microwave Properties of Microwires. Moldavian J Phys Sci 14(3-4): 201-214.

7. Baranov SA (1999) Generalized conductivity and optimum energy release. Tech Phys 44: 853-854.

8. Baranov SA (2017) An engineering review about microwire. Lambert (Academic publishing), pp: 1-42.

9. Baranov SA (2011) Magnetic models of cast amorphous microwires. Surface Engineering and Applied Electrochemistry 47(4): 316-330.

10. Baranov SA (2003) Magnetic properties of Co-based amorphous microwire. Journal of Magnetism and Magnetic Materials 266(3): 278-281.

11. Peng HX, Qin F, Phan MH (2016) Ferromagnetic microwires composites: From sensors to microwave applications. $1^{\text {st }}$ (Edn.), Springer International Publishing, Switzerland, pp: 245.

12. Qin F, Peng HX (2013) Ferromagnetic microwires enabled multifunctional composite materials. Progr Mater Sci 58(2): 183-259. 